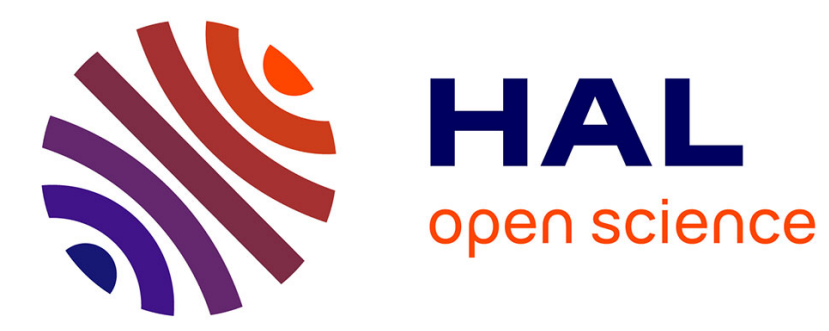

\title{
GEVREY REGULARITY FOR SOLUTION OF THE SPATIALLY HOMOGENEOUS LANDAU EQUATION
}

\author{
Hua Chen, Weixi Li, Chao-Jiang Xu
}

\section{To cite this version:}

Hua Chen, Weixi Li, Chao-Jiang Xu. GEVREY REGULARITY FOR SOLUTION OF THE SPATIALLY HOMOGENEOUS LANDAU EQUATION. Acta Mathematica Scientia, 2009, 29B (3), pp.673-686. hal-00434300

\section{HAL Id: hal-00434300 https://hal.science/hal-00434300}

Submitted on 22 Nov 2009

HAL is a multi-disciplinary open access archive for the deposit and dissemination of scientific research documents, whether they are published or not. The documents may come from teaching and research institutions in France or abroad, or from public or private research centers.
L'archive ouverte pluridisciplinaire HAL, est destinée au dépôt et à la diffusion de documents scientifiques de niveau recherche, publiés ou non, émanant des établissements d'enseignement et de recherche français ou étrangers, des laboratoires publics ou privés. 


\title{
GEVREY REGULARITY FOR THE SOLUTION OF THE SPATIALLY HOMOGENEOUS LANDAU EQUATION
}

\author{
HUA CHEN, WEI-XI LI AND CHAO-JIANG XU
}

\begin{abstract}
In this paper, we study the Gevrey class regularity for solutions of the spatially homogeneous Landau equations in the hard potential case and the Maxwellian molecules case.
\end{abstract}

\section{INTRODUCTION}

In this paper we study the smoothness effects of solutions for the following Cauchy problem of the spatially homogeneous Landau equation

$$
\left\{\begin{array}{l}
\partial_{t} f=\nabla_{v} \cdot\left\{\int_{\mathbb{R}^{3}} a\left(v-v_{*}\right)\left[f\left(v_{*}\right) \nabla_{v} f(v)-f(v) \nabla_{v} f\left(v_{*}\right)\right] d v_{*}\right\} \\
f(0, v)=f_{0}(v)
\end{array}\right.
$$

where $f(t, v) \geq 0$ stands for the density of particles with velocity $v \in \mathbb{R}^{3}$ at time $t \geq 0$, and $\left(a_{i j}\right)$ is a nonnegative symmetric matrix given by

$$
a_{i j}(v)=\left(\delta_{i j}-\frac{v_{i} v_{j}}{|v|^{2}}\right)|v|^{\gamma+2} .
$$

We only consider here the condition $\gamma \in[0,1]$. It's called the hard potential case when $\gamma \in] 0,1]$ and the Maxwellian molecules case when $\gamma=0$.

Set $c=\sum_{i, j=1}^{3} \partial_{v_{i} v_{j}} a_{i j}=-2(\gamma+3)|v|^{\gamma}$ and

$$
\bar{a}_{i j}(t, v)=\left(a_{i j} * f\right)(t, v)=\int_{\mathbb{R}^{3}} a_{i j}\left(v-v_{*}\right) f\left(t, v_{*}\right) d v_{*}, \quad \bar{c}=c * f .
$$

Then the Cauchy problem (1) can be rewritten as the following form,

$$
\left\{\begin{array}{l}
\partial_{t} f=\sum_{i, j=1}^{3} \bar{a}_{i j} \partial_{v_{i} v_{j}} f-\bar{c} f \\
f(0, v)=f_{0}(v) .
\end{array}\right.
$$

This is a non-linear diffusion equation, and the coefficients $\bar{a}_{i j}, \bar{c}$ depend on the solution $f$.

Here we are mainly concerned with the Gevrey class regularity for the solution of the Landau equation. This equation is obtained as a limit of the Boltzmann equation when the collisions become grazing (see [8] and references therein). Recently, a lot of progress has been made on the study of the Sobolev regularizing property, cf. [6, 11, 13, 18, 19] and references therein, which shows that in some sense the Landau equation can be regarded as a non-linear and non-local analog of the hypo-elliptic Fokker-Planck equation. That means the weak solution, which constructed under rather weak hypothesis on the initial datum, will become smooth or, even more, rapidly decreasing in $v$ at infinity. This behavior is quite similar to that of the spatially homogeneous Boltzmann equation without cut-off (see [12, 10] for more details).

2000 Mathematics Subject Classification. 35B65, 82B40.

Key words and phrases. Landau equation, Gevrey regularity, Cauchy problem. 
In the Gevrey class frame, some results have been obtained concerning the propagation property for solutions of the Boltzmann equation, e.g. the solutions having the Gevrey regularity have been constructed in [17] for initial data having the same Gevrey regularity, and the uniform propagation in all time of the Gevrey regularity has been proved in [9] in the case of Maxwellian molecules. Recently a general Gevrey regularity result have been given in [15] for spatially homogeneous and linear Boltzmann equation for any initial data.

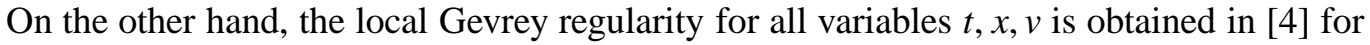
some semi-linear Fokker-Planck equations, which implies that, in this case, there are also the smoothness effects which is similar to the heat equation case.

Now we give some notations used throughout the paper. For a multi-index $\alpha=\left(\alpha_{1}, \alpha_{2}, \alpha_{3}\right)$, we denote $|\alpha|=\alpha_{1}+\alpha_{2}+\alpha_{3}, \alpha !=\alpha_{1} ! \alpha_{2} ! \alpha_{3} !$ and $\partial^{\alpha}=\partial_{v_{1}}^{\alpha_{1}} \partial_{v_{2}}^{\alpha_{2}} \partial_{v_{3}}^{\alpha_{3}}$. We say $\beta=\left(\beta_{1}, \beta_{2}, \beta_{3}\right) \leq$ $\left(\alpha_{1}, \alpha_{2}, \alpha_{3}\right)=\alpha$ if $\beta_{i} \leq \alpha_{i}$ for each $i$. Denote by $M(f(t)), E(f(t))$ and $H(f(t))$ respectively the mass, energy and entropy of the function $f(t)$, i.e.,

$$
\begin{gathered}
M(f(t))=\int_{\mathbb{R}^{3}} f(t, v) d v, \quad E(f(t))=\frac{1}{2} \int_{\mathbb{R}^{3}} f(t, v)|v|^{2} d v, \\
H(f(t))=\int_{\mathbb{R}^{3}} f(t, v) \log f(t, v) d v .
\end{gathered}
$$

and denote $M_{0}=M(f(0)), E_{0}=E(f(0))$ and $H_{0}=H(f(0))$. We know that the solution of the Landau equation satisfies the formal conservation laws:

$$
M(f(t))=M_{0}, \quad E(f(t))=E_{0}, \quad H(f(t)) \leq H_{0}, \quad \forall t \geq 0 .
$$

Also we adopt the following notations,

$$
\begin{gathered}
\left\|\partial^{\alpha} f(t, \cdot)\right\|_{L_{s}^{p}}^{p}=\left\|\partial^{\alpha} f(t)\right\|_{L_{s}^{p}}^{p}=\int_{\mathbb{R}^{3}}\left|\partial^{\alpha} f(t, v)\right|^{p}\left(1+|v|^{2}\right)^{s / 2} d v, \text { for } p \geq 1, \\
\|f(t, \cdot)\|_{H_{s}^{m}}^{2}=\|f(t)\|_{H_{s}^{m}}^{2}=\sum_{|\alpha| \leq m}\left\|\partial^{\alpha} f(t, \cdot)\right\|_{L_{s}^{2}}^{2} .
\end{gathered}
$$

Before stating our main result, let us recall the definition of the Gevrey class function space $G^{\sigma}\left(\mathbb{R}^{N}\right)$, where $\sigma \geq 1$ is the Gevrey index (cf. [5, 16]). Let $u$ be a real function defined in $\mathbb{R}^{N}$. We say $u \in G^{\sigma}\left(\mathbb{R}^{N}\right)$ if $u \in C^{\infty}\left(\mathbb{R}^{N}\right)$ and there exists a positive constant $C$ such that for all multi-indices $\alpha \in \mathbb{N}^{N}$, we have

$$
\left\|\partial^{\alpha} u\right\|_{L^{2}\left(\mathbb{R}^{3}\right)} \leq C^{|\alpha|+1}(|\alpha| !)^{\sigma} .
$$

We denote by $G_{0}^{\sigma}\left(\mathbb{R}^{3}\right)$ the space of Gevrey functions with compact support. Note that $G^{1}\left(\mathbb{R}^{N}\right)$ is the space of real analytic functions.

In the hard potential case, the existence, uniqueness and Sobolev regularity of the weak solution had been studied by Desvillettes and Villani (see [11], Theorem 5, Theorem 6 and Theorem 7). Actually they proved that, under rather weak assumptions on the initial datum (for instance if $f_{0} \in L_{2+\delta}^{1}$ with $\delta>0$ ), there exists a weak solution $f$ of the Cauchy problem (3) such that for all time $t_{0}>0$, all integer $m \geq 0$, and all $s>0$,

$$
\sup _{t \geq t_{0}}\|f(t, \cdot)\|_{H_{s}^{m}} \leq C,
$$

where $C$ is a constant depending only on $\gamma, M_{0}, E_{0}, H_{0}, m, s$ and $t_{0}$. Furthermore they proved that $f(t, v) \in C^{\infty}\left(\mathbb{R}_{t}^{+} ; \mathcal{S}\left(\mathbb{R}_{v}^{3}\right)\right)$, where $\left.\mathbb{R}_{t}^{+}=\right] 0,+\infty\left[\right.$ and $\mathcal{S}\left(\mathbb{R}_{v}^{3}\right)$ denotes the space of smooth functions rapidly decreasing at infinity. If $f_{0} \in L_{p}^{2}$ with $p>5 \gamma+15$, then the Cauchy problem admits a unique smooth solution . 
In the Maxwellian case, Villani [19] proved that the Cauchy problem admits a unique classical solution $f$ for any initial datum and for all $t>0$, and that $f$ is bounded and lies in $C^{\infty}\left(\mathbb{R}_{v}^{3}\right)$.

Now starting from the smooth solution, we state our main result on the Gevrey regularity as follows.

Theorem 1.1. Let $f_{0}$ be the initial datum with finite mass, energy and entropy and $f$ be any solution of the Cauchy problem (3) such that for all $t_{0}$, $t_{1}$ with $0<t_{0}<t_{1}<+\infty$, and all integer $m \geq 0$,

$$
\sup _{t \in\left[t_{0}, t_{1}\right]}\|f(t, \cdot)\|_{H_{\gamma}^{m}}<+\infty .
$$

Then for any number $\sigma>1$, we have $f(t, \cdot) \in G^{\sigma}\left(\mathbb{R}^{3}\right)$ for all time $t>0$.

Remark 1.2. Our result, which is given here for the space dimension to be equal to 3, will also be true for any space dimension.

This paper includes three sections. The proof of the main result Theorem 1.1 will be given in Section 2 and in Section 3 we shall mainly prove Lemma 2.2, which is crucial in the proof of Section 2

\section{Proof of the Main Results}

This section is devoted to the proof of the main result. In the sequel, we always use $\sum_{1 \leq|\beta| \leq|\mu|}$ to denotes the summation over all the multi-indices $\beta$ satisfying $\beta \leq \mu$ and $1 \leq$ $|\beta| \leq|\mu|$. Likewise $\sum_{1 \leq|\beta| \leq|\mu|-1}$ denotes the summation over all the multi-indices $\beta$ satisfying $\beta \leq \mu$ and $1 \leq|\beta| \leq|\mu|-1$. Firstly we have

Lemma 2.1. For any $\sigma>1$, there exists a constant $C_{\sigma}$, depending only on $\sigma$, such that for all multi-indices $\mu \in \mathbb{N}^{3},|\mu| \geq 1$,

$$
\sum_{1 \leq|\beta| \leq|\mu|} \frac{1}{|\beta|^{3}} \leq C_{\sigma}|\mu|^{\sigma-1}
$$

and

$$
\sum_{1 \leq|\beta| \leq|\mu|-1} \frac{1}{|\beta|^{2}(|\mu|-|\beta|)} \leq C_{\sigma}|\mu|^{\sigma-1} .
$$

Proof For each positive integer $l$, we denote by $N\{|\beta|=l\}$ the number of the multiindices $\beta$ with $|\beta|=l$. In the case when the space dimension equals to 3 , one has

$$
N\{|\beta|=l\}=\frac{(l+2) !}{2 ! l !}=\frac{1}{2}(l+1)(l+2) .
$$

It's easy to see that

$$
\sum_{1 \leq\langle\beta|\leq| \mu|} \frac{1}{|\beta|^{3}} \leq \sum_{l=1}^{|\mu|} \sum_{|\beta|=l} \frac{1}{l^{3}}=\sum_{l=1}^{|\mu|} \frac{N\{|\beta|=l\}}{l^{3}}
$$

We combine these estimates to compute

$$
\sum_{1 \leq|\beta| \leq|\mu|} \frac{1}{|\beta|^{3}} \leq \frac{1}{2} \sum_{l=1}^{|\mu|} \frac{(l+1)(l+2)}{l^{3}} \leq 3 \sum_{l=1}^{|\mu|} \frac{1}{l} .
$$


Together with the fact that $3 \sum_{l=1}^{|\mu|} l^{-1} \leq C_{\sigma}|\mu|^{\sigma-1}$ for some constant $C_{\sigma}$, this gives the estimate (5). In a similar way as above we can prove the estimate (6). The proof is completed.

The following lemma, which will be proved in the next section, is of great of use to us. For simplicity, in the following discussions, we shall use the notation $\gamma-j$, with $\gamma$ a multiindex and $j$ an integer, to denotes some multi-index $\tilde{\gamma}$ satisfying $\tilde{\gamma} \leq \gamma$ and $|\tilde{\gamma}|=|\mu|-j$.

Lemma 2.2. Let $\sigma>1$. There exist constants $B, C_{1}, C_{2}>0$ with $B$ depending only on the Gevrey index $\sigma$ and $C_{1}, C_{2}$ depending only on $M_{0}, E_{0}, H_{0}, \sigma$ and $\gamma$, such that for all multi-indices $\mu \in \mathbb{N}^{3}$ with $|\mu| \geq 2$ and all $t>0$, we have

$$
\begin{aligned}
\frac{d}{d t} & \|\| \partial^{\mu} f(t)_{L^{2}}^{2}+C_{1}\left\|\nabla_{\nu} \partial^{\mu} f(t)\right\|_{L_{\gamma}^{2}}^{2} \leq C_{2}|\mu|^{2}\left\|\nabla_{\nu} \partial^{\mu-1} f(t)\right\|_{L_{\gamma}^{2}}^{2} \\
& +C_{2} \sum_{2 \leq|\beta| \leq|\mu|} C_{\mu}^{\beta}\left\|\nabla_{\nu} \partial^{\mu-\beta+1} f(t)\right\|_{L_{\gamma}^{2}} \cdot\left\|\nabla_{\nu} \partial^{\mu-1} f(t)\right\|_{L_{\gamma}^{2}} \cdot\left[G_{\sigma}(f(t))\right]_{\beta-2} \\
& +C_{2} \sum_{0 \leq|\beta| \leq|\mu|} C_{\mu}^{\beta}\left\|\partial^{\beta} f(t)\right\|_{L_{\gamma}^{2}} \cdot\left\|\nabla_{\nu} \partial^{\mu-1} f(t)\right\|_{L_{\gamma}^{2}} \cdot\left[G_{\sigma}(f(t))\right]_{\mu-\beta},
\end{aligned}
$$

where $C_{\mu}^{\beta}=\frac{\mu !}{(\mu-\beta) ! \beta !}$ is the binomial coefficients, and

$$
\left[G_{\sigma}(f(t))\right]_{\beta-2}=\left\|\partial^{\beta-2} f(t)\right\|_{L^{2}}+B^{|\beta|-2}((|\beta|-2) !)^{\sigma} .
$$

From now on, $\Omega$ will be used to denote an arbitrary fixed interval $\left[T_{0}, T_{1}\right]$ with $0<T_{0}<$ $T_{1}<T_{0}+1$. We denote

$$
\Omega_{\rho}=\left[T_{0}+\rho, T_{1}-\rho\right], \quad 0<\rho<\frac{T_{1}-T_{0}}{2}<\frac{1}{2} .
$$

For any integer $k$ with $k \geq 2$ and any $\rho$ with $0<\rho<\left(T_{1}-T_{0}\right) / 2$, take a function $\varphi_{\rho, k}(t) \in C_{0}^{\infty}(\mathbb{R})$ satisfying $0 \leq \varphi_{\rho, k} \leq 1$, and $\operatorname{Supp} \varphi_{\rho, k} \subset \Omega_{\tilde{\rho}}$ with $\tilde{\rho}=\frac{k-1}{k} \rho$, and $\varphi_{\rho, k}=1$ in $\Omega_{\rho}$. it is easy to verify

$$
\sup \left|\frac{d^{j} \varphi_{\rho, k}}{d t^{j}}\right| \leq \tilde{C}_{j}(k / \rho)^{j}, \quad \forall j \in \mathbb{N} .
$$

And for $\tilde{\rho}=(k-1) \rho / k, k \geq 2$, the following simple fact is clear,

$$
\frac{1}{\rho^{k}} \leq \frac{1}{\tilde{\rho}^{k}}=\frac{1}{\rho^{k}} \times\left(\frac{k}{k-1}\right)^{k} \leq \frac{5}{\rho^{k}} .
$$

Now we are prepare to prove the main results, which can be deduced easily from the following

Proposition 2.3. Let $f_{0}$ be the initial datum with finite mass, energy and entropy and $f$ be any solution of the Cauchy problem satisfying (\#). Then for $\sigma>1$, there exists a constant $A$, depending only on $T_{0}, T_{1}, M_{0}, E_{0}, H_{0}, \gamma$ and $\sigma$, such that for any $k \in \mathbb{N}, k \geq 0$,

$$
(Q)_{k} \quad \sup _{t \in \Omega_{\rho}}\left\|\partial^{\alpha} f(t)\right\|_{L^{2}}+\left\{\int_{T_{0}+\rho}^{T_{1}-\rho}\left\|\nabla_{\nu} \partial^{\tilde{\alpha}} f(t)\right\|_{L_{\gamma}^{2}}^{2} d t\right\}^{1 / 2} \leq \frac{A^{k}}{\rho^{k}}[(k-1) !]^{\sigma}
$$

holds for any multi-indices $\alpha, \tilde{\alpha}$ with $|\alpha|=|\tilde{\alpha}|=k$ and all $\rho$ with $0<\rho<\left(T_{1}-T_{0}\right) / 2$. Here we assume $(-1) !=0 !=1$.

Proof We use induction on $k$. $(Q)_{0},(Q)_{1}$ obviously hold if we take $A$ large enough such that

$$
A \geq \sup _{s \in\left[T_{0}, T_{1}\right]}\|f(s)\|_{H_{\gamma}^{2}}+1
$$


The term on the right hand of (9) is finite by virtue of (4). Now assuming $(Q)_{k-1}$ holds, we shall show the truth of $(Q)_{k}, k \geq 2$. In this proof $C_{j}, j \geq 3$, are used to denote different constants depending only on $T_{0}, T_{1}, M_{0}, E_{0}, H_{0}, \gamma$ and $\sigma$.

Firstly we shall prove

$$
\sup _{t \in \Omega_{\rho}}\left\|\partial^{\alpha} f(t)\right\|_{L^{2}} \leq \frac{1}{2} \frac{A^{|\alpha|}}{\rho^{|\alpha|}}[(|\alpha|-1) !]^{\sigma}, \quad \forall|\alpha|=k, \quad \forall 0<\rho<\frac{T_{1}-T_{2}}{2} .
$$

In the following discussion, let $\alpha$ be any multi-index with $|\alpha|=k$ and $\rho$ be any number with $0<\rho<\left(T_{1}-T_{2}\right) / 2$. Applying Lemma 2.2 with $\mu=\alpha$, we obtain

$$
\begin{aligned}
& \frac{d}{d t} \quad\left\|\partial^{\alpha} f(t)\right\|_{L^{2}}^{2}+C_{1}\left\|\nabla_{\nu} \partial^{\alpha} f(t)\right\|_{L_{\gamma}^{2}}^{2} \leq C_{2}|\alpha|^{2}\left\|\nabla_{\nu} \partial^{\alpha-1} f(t)\right\|_{L_{\gamma}^{2}}^{2} \\
& +\quad C_{2} \sum_{2 \leq|\beta| \leq|\alpha|} C_{\alpha}^{\beta}\left\|\nabla_{\nu} \partial^{\alpha-\beta+1} f(t)\right\|_{L_{\gamma}^{2}} \cdot\left\|\nabla_{\nu} \partial^{\alpha-1} f(t)\right\|_{L_{\gamma}^{2}} \cdot\left[G_{\sigma}(f(t))\right]_{\beta-2} \\
& +\quad C_{2} \sum_{0 \leq|\beta| \leq|\alpha|} C_{\alpha}^{\beta}\left\|\partial^{\beta} f(t)\right\|_{L_{\gamma}^{2}} \cdot\left\|\nabla_{\nu} \partial^{\alpha-1} f(t)\right\|_{L_{\gamma}^{2}} \cdot\left[G_{\sigma}(f(t))\right]_{\alpha-\beta} .
\end{aligned}
$$

Write the last term of the right side as

$$
\begin{gathered}
C_{2}\|f(t)\|_{L_{\gamma}^{2}} \cdot\left\|\nabla_{\nu} \partial^{\alpha-1} f(t)\right\|_{L_{\gamma}^{2}} \cdot\left[G_{\sigma}(f(t))\right]_{\alpha} \\
+C_{2} \sum_{1 \leq \beta|\leq| \alpha \mid} C_{\alpha}^{\beta}\left\|\partial^{\beta} f(t)\right\|_{L_{\gamma}^{2}} \cdot\left\|\nabla_{\nu} \partial^{\alpha-1} f(t)\right\|_{L_{\gamma}^{2}} \cdot\left[G_{\sigma}(f(t))\right]_{\alpha-\beta} .
\end{gathered}
$$

And then we get

$$
\begin{aligned}
\frac{d}{d t} \quad & \left\|\partial^{\alpha} f(t)\right\|_{L^{2}}^{2}+C_{1}\left\|\nabla_{\nu} \partial^{\alpha} f(t)\right\|_{L_{\gamma}^{2}}^{2} \leq C_{2}|\alpha|^{2}\left\|\nabla_{\nu} \partial^{\alpha-1} f(t)\right\|_{L_{\gamma}^{2}}^{2} \\
+ & C_{2}\|f(t)\|_{L_{\gamma}^{2}} \cdot\left\|\nabla_{\nu} \partial^{\alpha-1} f(t)\right\|_{L_{\gamma}^{2}} \cdot\left[G_{\sigma}(f(t))\right]_{\alpha} \\
+ & C_{2} \sum_{2 \leq|\beta| \leq|\alpha|} C_{\alpha}^{\beta}\left\|\nabla_{\nu} \partial^{\alpha-\beta+1} f(t)\right\|_{L_{\gamma}^{2}} \cdot\left\|\nabla_{\nu} \partial^{\alpha-1} f(t)\right\|_{L_{\gamma}^{2}} \cdot\left[G_{\sigma}(f(t))\right]_{\beta-2} \\
+ & C_{2} \sum_{1 \leq|\beta| \leq|\alpha|} C_{\alpha}^{\beta}\left\|\partial^{\beta} f(t)\right\|_{L_{\gamma}^{2}} \cdot\left\|\nabla_{\nu} \partial^{\alpha-1} f(t)\right\|_{L_{\gamma}^{2}} \cdot\left[G_{\sigma}(f(t))\right]_{\alpha-\beta} .
\end{aligned}
$$

Multiplying by $\varphi_{\rho, k}(t)$ the both sides of the above inequality, one has

$$
\begin{array}{ll}
\frac{d}{d t} \quad\left[\varphi_{\rho, k}(t)\left\|\partial^{\alpha} f(t)\right\|_{L^{2}}^{2}\right]+C_{1} \varphi_{\rho, k}(t)\left\|\nabla_{\nu} \partial^{\alpha} f(t)\right\|_{L_{\gamma}^{2}}^{2} \\
\leq \quad \frac{d \varphi_{\rho, k}}{d t} \cdot\left\|\partial^{\alpha} f(t)\right\|_{L^{2}}^{2}+C_{2} \cdot \varphi_{\rho, k}(t)|\alpha|^{2}\left\|\nabla_{\nu} \partial^{\alpha-1} f(t)\right\|_{L_{\gamma}^{2}}^{2} \\
+\quad C_{2} \cdot \varphi_{\rho, k}(t)\|f(t)\|_{L_{\gamma}^{2}} \cdot\left\|\nabla_{\nu} \partial^{\alpha-1} f(t)\right\|_{L_{\gamma}^{2}} \cdot\left[G_{\sigma}(f(t))\right]_{\alpha} \\
+\quad C_{2} \cdot \varphi_{\rho, k}(t) \sum_{2 \leq|\beta| \leq|\alpha|} C_{\alpha}^{\beta}\left\|\nabla_{\nu} \partial^{\alpha-\beta+1} f(t)\right\|_{L_{\gamma}^{2}} \cdot\left\|\nabla_{\nu} \partial^{\alpha-1} f(t)\right\|_{L_{\gamma}^{2}} \cdot\left[G_{\sigma}(f(t))\right]_{\beta-2} \\
+\quad C_{2} \cdot \varphi_{\rho, k}(t) \sum_{1 \leq|\beta| \leq|\alpha|} C_{\alpha}^{\beta}\left\|\partial^{\beta} f(t)\right\|_{L_{\gamma}^{2}} \cdot\left\|\nabla_{\nu} \partial^{\alpha-1} f(t)\right\|_{L_{\gamma}^{2}} \cdot\left[G_{\sigma}(f(t))\right]_{\alpha-\beta} .
\end{array}
$$

To simplify the notation, we set

$$
\left[G_{\sigma}(f)\right]_{\rho, \beta}=\sup _{s \in \Omega_{\rho}}\left[G_{\sigma}(f(s))\right]_{\beta}=\sup _{s \in \Omega_{\rho}}\left\|\partial^{\beta} f(s)\right\|_{L^{2}}+B^{|\beta|}(|\beta| !)^{\sigma} .
$$

Recall Supp $\varphi_{\rho, k} \subset \Omega_{\tilde{\rho}}$ with $\tilde{\rho}=(k-1) \rho / k$ and $\varphi_{\rho, k}(t)=1$ for all $t \in \Omega_{\rho}$ and $\varphi_{\rho, k}\left(T_{0}\right)=0$. Then for any $t \in \Omega_{\rho}$, we integrate the above inequality over the interval $\left[T_{0}, t\right] \subset\left[T_{0}, T_{1}-\rho\right]$ 
and then use Cauchy inequality to get

$$
\begin{array}{ll} 
& \left\|\partial^{\alpha} f(t)_{L^{2}}^{2}=\varphi_{\rho, k}(t)\right\| \partial^{\alpha} f(t)\left\|_{L^{2}}^{2}-\varphi_{\rho, k}\left(T_{0}\right)\right\| \partial^{\alpha} f\left(T_{0}\right) \|_{L^{2}}^{2} \\
\leq & \sup \left|\frac{d \varphi_{\rho, k}}{d t}\right| \int_{T_{0}+\tilde{\rho}}^{T_{1}-\tilde{\rho}}\left\|\partial^{\alpha} f(s)\right\|_{L^{2}}^{2} d s+C_{2}|\alpha|^{2} \int_{T_{0}+\tilde{\rho}}^{T_{1}-\tilde{\rho}}\left\|\nabla_{\nu} \partial^{\alpha-1} f(s)\right\|_{L_{\gamma}^{2}}^{2} d s \\
+ & C_{2} \sup _{s \in \Omega_{\tilde{\rho}}}\|f(s)\|_{L_{\gamma}^{2}} \cdot\left\{\int_{T_{0}+\tilde{\rho}}^{T_{1}-\tilde{\rho}}\left[G_{\sigma}(f(s))\right]_{\alpha}^{2} d s\right\}^{\frac{1}{2}}\left\{\int_{T_{0}+\tilde{\rho}}^{T_{1}-\tilde{\rho}}\left\|\nabla_{\nu} \partial^{\alpha-1} f(s)\right\|_{L_{\gamma}^{2}}^{2} d s\right\}^{\frac{1}{2}} \\
+ & C_{2} \sum_{2 \leq|\beta| \leq|\alpha|} C_{\alpha}^{\beta}\left[G_{\sigma}(f)\right]_{\tilde{\rho}, \beta-2}\left\{\int_{T_{0}+\tilde{\rho}}^{T_{1}-\tilde{\rho}}\left\|\nabla_{\nu} \partial^{\alpha-\beta+1} f(s)\right\|_{L_{\gamma}^{2}}^{2} d s\right\}^{\frac{1}{2}} \\
& \times \quad\left\{\int_{T_{0}+\tilde{\rho}}^{T_{1}-\tilde{\rho}}\left\|\nabla_{\nu} \partial^{\alpha-1} f(s)\right\|_{L_{\gamma}^{2}}^{2} d s\right\}^{\frac{1}{2}} \\
+ & C_{2} \sum_{1 \leq|\beta| \leq|\alpha|} C_{\alpha}^{\beta}\left[G_{\sigma}(f)\right]_{\tilde{\rho}, \alpha-\beta}\left\{\int_{T_{0}+\tilde{\rho}}^{T_{1}-\tilde{\rho}}\left\|\partial^{\beta} f(s)\right\|_{L_{\gamma}^{2}}^{2} d s\right\}^{\frac{1}{2}}\left\{\int_{T_{0}+\tilde{\rho}}^{T_{1}-\tilde{\rho}}\left\|\nabla_{\nu} \partial^{\alpha-1} f(s)\right\|_{L_{\gamma}^{2}}^{2} d s\right\}^{\frac{1}{2}} \\
= & \left(S_{1}\right)+\left(S_{2}\right)+\left(S_{3}\right)+\left(S_{4}\right)+\left(S_{5}\right) .
\end{array}
$$

In order to treat the above five terms, we need the following estimates which are deduced directly from the the induction hypothesis. By the truth of $(Q)_{k-1}$, we have

$$
\begin{gathered}
\left\{\int_{T_{0}+\tilde{\rho}}^{T_{1}-\tilde{\rho}}\left\|\nabla_{\nu} \partial^{\alpha-1} f(s)\right\|_{L_{\gamma}^{2}}^{2} d s\right\}^{1 / 2} \leq \frac{A^{|\alpha|-1}}{\tilde{\rho}^{|\alpha|-1}}[(|\alpha|-2) !]^{\sigma} ; \\
\left\{\int_{T_{0}+\tilde{\rho}}^{T_{1}-\tilde{\rho}}\left\|\nabla_{\nu} \partial^{\alpha-\beta+1} f(s)\right\|_{L_{\gamma}^{2}}^{2} d s\right\}^{\frac{1}{2}} \leq \frac{A^{|\alpha|-|\beta|+1}}{\tilde{\rho}^{|\alpha|-\beta \mid+1}}[(|\alpha|-|\beta|) !]^{\sigma}, 2 \leq|\beta| \leq|\alpha| ;
\end{gathered}
$$

and

$$
\left\{\int_{T_{0}+\tilde{\rho}}^{T_{1}-\tilde{\rho}}\left\|\partial^{\beta} f(s)\right\|_{L_{\gamma}^{2}}^{2} d s\right\}^{1 / 2} \leq \frac{A^{|\beta|-1}}{\tilde{\rho}^{|\beta|-1}}[(|\beta|-2) !]^{\sigma}, \quad 2 \leq|\beta| \leq|\alpha| .
$$

the last inequality using the fact $\left\|\partial^{\beta} f\right\|_{L_{\gamma}^{2}} \leq\left\|\nabla_{\nu} \partial^{\beta-1} f\right\|_{L_{\gamma}^{2}}$ for any $\beta$ with $2 \leq|\beta| \leq \alpha$. Observe that there exists a constant $\tilde{B}>1$, depending only on $B$ and $\sigma$, such that

$$
\left\{\begin{array}{l}
B^{m}(m !)^{\sigma} \leq \tilde{B}^{m}((m-1) !)^{\sigma}, \quad 1 \leq m \leq|\alpha|-1, \\
B^{|\alpha|}(|\alpha| !)^{\sigma} \leq \tilde{B}^{|\alpha|-1}((|\alpha|-2) !)^{\sigma}
\end{array}\right.
$$

With (13), one has, by taking $A$ such that $A \geq \tilde{B}$,

$$
\left\{\int_{T_{0}+\tilde{\rho}}^{T_{1}-\tilde{\rho}}\left[G_{\sigma}(f(s))\right]_{\alpha}^{2} d s\right\}^{1 / 2} \leq \frac{2 A^{|\alpha|-1}}{\tilde{\rho}^{|\alpha|-1}}[(|\alpha|-2) !]^{\sigma} .
$$

Next we shall treat the term $\left[G_{\sigma}(f)\right]_{\tilde{\rho}, \lambda}$ which equals to $\sup _{s \in \Omega_{\tilde{\rho}}}\left\|\partial^{\lambda} f(s)\right\|_{L^{2}}+B^{|\lambda|}(|\lambda| !)^{\sigma}$ by definition. It follows from the truth of $(Q)_{k-1}$ again that

$$
\sup _{t \in \Omega_{\tilde{\rho}}}\left\|\partial^{\lambda} f(t)\right\|_{L^{2}} \leq \frac{A^{|\lambda|}}{\tilde{\rho}^{|\lambda|}}((|\lambda|-1) !)^{\sigma}, \quad \forall \lambda, 1 \leq|\lambda| \leq k-1,
$$

from which and the fact $A \geq \tilde{B}$, we get the estimate on $\left[G_{\sigma}(f)\right]_{\tilde{\rho}, \lambda}$, that is,

$$
\left[G_{\sigma}(f)\right]_{\tilde{\rho}, \lambda} \leq \frac{2 A^{|\lambda|}}{\tilde{\rho}^{|\lambda|}}((|\lambda|-1) !)^{\sigma}, \quad 1 \leq|\lambda| \leq|\alpha|-1 .
$$


Now the above estimates allow us to deal with the terms from $\left(S_{1}\right)$ to $\left(S_{5}\right)$. Note that $\left\|\partial^{\alpha} f(s)\right\|_{L^{2}} \leq\left\|\partial^{\alpha} f(s)\right\|_{L_{\gamma}^{2}}$ and hence from (7) and (13), it follows immediately that

$$
\left(S_{1}\right) \leq \frac{C_{3} k}{\rho}\left\{\frac{A^{|\alpha|-1}}{\tilde{\rho}^{|\alpha|-1}}[(|\alpha|-2) !]^{\sigma}\right\}^{2} .
$$

This along with the facts $k=|\alpha|$ and $\rho^{-1}<\tilde{\rho}^{-1}<\tilde{\rho}^{-2}$ shows at once

$$
\left(S_{1}\right) \leq C_{4}\left\{\frac{A^{|\alpha|-1}}{\tilde{\rho}^{|\alpha|}}[(|\alpha|-1) !]^{\sigma}\right\}^{2}
$$

And by virtue of (11), we obtain

$$
\left(S_{2}\right) \leq C_{2}|\alpha|^{2}\left\{\frac{A^{|\alpha|-1}}{\tilde{\rho}^{|\alpha|-1}}[(|\alpha|-2) !]^{\sigma}\right\}^{2} \leq C_{5}\left\{\frac{A_{\tilde{\rho}}^{|\alpha|-1}}{\tilde{\rho}^{|\alpha|-1}}[(|\alpha|-1) !]^{\sigma}\right\}^{2} .
$$

For the term $\left(S_{3}\right)$, Combining (9), (11) and (14), one has

$$
\left(S_{3}\right) \leq C_{6} A\left\{\frac{A^{|\alpha|-1}}{\tilde{\rho}^{|\alpha|-1}}[(|\alpha|-2) !]^{\sigma}\right\}^{2} .
$$

The treatment of the terms $\left(S_{4}\right)$ is a little more complicated. Write $\left(S_{4}\right)=\left(S_{4}\right)^{\prime}+\left(S_{4}\right)^{\prime \prime}$ with

$$
\left(S_{4}\right)^{\prime}=C_{2} \sum_{|\beta|=2} C_{\alpha}^{\beta}\left[G_{\sigma}(f)\right]_{\tilde{\rho}, 0}\left\{\int_{T_{0}+\tilde{\rho}}^{T_{1}-\tilde{\rho}}\left\|\nabla_{v} \partial^{\alpha-\beta+1} f(s)\right\|_{L_{\gamma}^{2}}^{2} d s\right\}^{\frac{1}{2}}\left\{\int_{T_{0}+\tilde{\rho}}^{T_{1}-\tilde{\rho}}\left\|\nabla_{v} \partial^{\alpha-1} f(s)\right\|_{L_{\gamma}^{2}}^{2} d s\right\}^{\frac{1}{2}}
$$

and

$$
\begin{aligned}
\left(S_{4}\right)^{\prime \prime}= & C_{2} \sum_{3 \leq|\beta| \leq|\alpha|} C_{\alpha}^{\beta}\left[G_{\sigma}(f)\right]_{\tilde{\rho}, \beta-2}\left\{\int_{T_{0}+\tilde{\rho}}^{T_{1}-\tilde{\rho}}\left\|\nabla_{\nu} \partial^{\alpha-\beta+1} f(s)\right\|_{L_{\gamma}^{2}}^{2} d s\right\}^{\frac{1}{2}} \\
& \times\left\{\int_{T_{0}+\tilde{\rho}}^{T_{1}-\tilde{\rho}}\left\|\nabla_{\nu} \partial^{\alpha-1} f(s)\right\|_{L_{\gamma}^{2}}^{2} d s\right\}^{\frac{1}{2}} .
\end{aligned}
$$

It is easy to verify that, by (9), (11) and (12),

$$
\left(S_{4}\right)^{\prime} \leq C_{7} A|\alpha|^{2}\left\{\frac{A^{|\alpha|-1}}{\tilde{\rho}^{|\alpha|-1}}[(|\alpha|-2) !]^{\sigma}\right\}^{2} \leq C_{8} A\left\{\frac{A^{|\alpha|-1}}{\tilde{\rho}^{|\alpha|-1}}[(|\alpha|-1) !]^{\sigma}\right\}^{2} .
$$

And by virtue of (11) and (15), we know $\left(S_{4}\right)^{\prime \prime}$ is bounded from above by

$$
\sum_{3 \leq|\beta| \leq|\alpha|} \frac{C_{3}|\alpha| !}{|\beta| !(|\alpha|-|\beta|) !} \frac{A^{|\beta|-2}}{\tilde{\rho}|\beta|-2}[(|\beta|-3) !]^{\sigma} \frac{A^{|\alpha|}}{\tilde{\rho}^{|\alpha|-\beta \beta \mid+1}}[(|\alpha|-|\beta|) !]^{\sigma} \frac{A^{|\alpha|-1}}{\tilde{\rho}^{|\alpha|-1}}[(|\alpha|-2) !]^{\sigma},
$$

from which we get

$$
\left(S_{4}\right)^{\prime \prime} \leq C_{9}\left\{\frac{A^{|\alpha|-1}}{\tilde{\rho}^{|\alpha|-1}}\right\}^{2}[(|\alpha|-2) !]^{\sigma} \sum_{3 \leq\langle\beta|\leq| \alpha|} \frac{|\alpha| !}{|\beta| !(|\alpha|-|\beta|) !}[(|\beta|-3) !]^{\sigma}[(|\alpha|-|\beta|) !]^{\sigma} .
$$

Direct verification shows that

$$
\begin{aligned}
\sum_{3 \leq|\beta| \leq|\alpha|} \frac{|\alpha| !}{|\beta| !(|\alpha|-|\beta|) !}[(|\beta|-3) !]^{\sigma}[(|\alpha|-|\beta|) !]^{\sigma} & \leq[(|\alpha|-1) !]^{\sigma} \sum_{3 \leq|\beta| \leq|\alpha|} \frac{6|\alpha|}{\left.\beta \beta\right|^{3}} \\
& \leq C_{10}[(|\alpha|-1) !]^{\sigma}|\alpha|^{\sigma} \quad(\operatorname{by}(5)) .
\end{aligned}
$$


Combining these, we get the estimate of $\left(S_{4}\right)^{\prime \prime}$; that is

$$
\left(S_{4}\right)^{\prime \prime} \leq C_{11}\left\{\frac{A^{|\alpha|-1}}{\tilde{\rho}^{|\alpha|-1}}[(|\alpha|-1) !]^{\sigma}\right\}^{2} .
$$

This with the estimate of $\left(S_{4}\right)^{\prime}$ gives

$$
\left(S_{4}\right)=\left(S_{4}\right)^{\prime}+\left(S_{4}\right)^{\prime \prime} \leq C_{12} A\left\{\frac{A^{|\alpha|-1}}{\tilde{\rho}^{|\alpha|-1}}[(|\alpha|-1) !]^{\sigma}\right\}^{2} .
$$

The term $\left(S_{5}\right)$ can be handled exactly as above, and we have, by virtue of (6), (9), (11) and (13),

$$
\left(S_{5}\right) \leq C_{13} A\left\{\frac{A^{|\alpha|-1}}{\tilde{\rho}^{|\alpha|-1}}[(|\alpha|-1) !]^{\sigma}\right\}^{2} .
$$

Now combination of (16), (17), (18), (19) and (20) gives that, for all $t \in \Omega \rho$,

$$
\left\|\partial^{\alpha} f(t)\right\|_{L^{2}}^{2} \leq\left(S_{1}\right)+\left(S_{2}\right)+\left(S_{3}\right)+\left(S_{4}\right)+\left(S_{5}\right) \leq C_{14} A\left\{\frac{A^{|\alpha|-1}}{\tilde{\rho}^{|\alpha|}}[(|\alpha|-1) !]^{\sigma}\right\}^{2} .
$$

Note that $\tilde{\rho}^{-|\alpha|}=\tilde{\rho}^{-k} \leq 5 \rho^{-|\alpha|}$ by (8), and hence the above inequality yields

$$
\left\|\partial^{\alpha} f(t)\right\|_{L^{2}}^{2} \leq C_{15} A\left\{\frac{A^{|\alpha|-1}}{\rho^{|\alpha|}}[(|\alpha|-1) !]^{\sigma}\right\}^{2}, \quad \forall t \in \Omega \rho .
$$

Taking $A$ large enough such that $A \geq 16 \max \left\{\sup _{s \in\left[T_{0}, T_{1}\right]}\|f(s)\|_{H_{\gamma}^{2}}+1, \tilde{B}, C_{15}\right\}$, then we get finally

$$
\left\|\partial^{\alpha} f(t)\right\|_{L^{2}}^{2} \leq\left\{\frac{1}{2} \frac{A^{|\alpha|}}{\rho^{|\alpha|}}[(|\alpha|-1) !]^{\sigma}\right\}^{2} .
$$

The above inequality holds for all $t \in \Omega_{\rho}$, and hence (10) follows.

In order to finish the proof, it remains to prove

$$
\left\{\int_{T_{0}+\rho}^{T_{1}-\rho}\left\|\nabla_{\nu} \partial^{\tilde{\alpha}} f(t)\right\|_{L_{\gamma}^{2}}^{2} d t\right\}^{\frac{1}{2}} \leq \frac{1}{2} \frac{A^{|\alpha|}}{\rho^{|\alpha|}}[(|\alpha|-1) !]^{\sigma}, \quad \forall|\tilde{\alpha}|=k, \quad \forall 0<\rho<\frac{T_{1}-T_{0}}{2} .
$$

And it can be handled exactly as (10). The only difference is that the multi-index $\alpha$ and the term $\left\|\partial^{\alpha} f(t)\right\|_{L^{2}}^{2}=\varphi_{\rho, k}(t)\left\|\partial^{\alpha} f(t)\right\|_{L^{2}}^{2}-\varphi_{\rho, k}\left(T_{0}\right)\left\|\partial^{\alpha} f\left(T_{0}\right)\right\|_{L^{2}}^{2}$ appearing in the above argument will be replace respectively by $\tilde{\alpha}$ and

$$
C_{1} \int_{T_{0}+\rho}^{T_{1}-\rho}\left\|\nabla_{\nu} \partial^{\tilde{\alpha}} f(s)\right\|_{L_{\gamma}^{2}}^{2} d s \leq C_{1} \int_{T_{0}+\tilde{\rho}}^{T_{1}-\tilde{\rho}} \varphi_{\rho, k}(s)\left\|\nabla_{\nu} \partial^{\tilde{\alpha}} f(s)\right\|_{L_{\gamma}^{2}}^{2} d s .
$$

Finally, combination of (10) and (21) gives the truth of $(Q)_{k}$. This completes the proof of Proposition 2.3.

\section{Proof of Lemma 2.2}

For simplicity , in this section, $\partial_{v_{i} v_{j}} \bar{a}_{i j}$ and $\bar{a}_{i j}$ will stand for $\sum_{1 \leq i, j \leq 3} \partial_{v_{i} v_{j}} \bar{a}_{i j}$ and $\sum_{i, j} \bar{a}_{i j}$, respectively. In the sequel $C$ is used to denote different constants which can be replaced by a larger one and depend only on $\gamma$, the Gevrey index $\sigma$, and the initial mass $M_{0}$, the initial energy $E_{0}$ and the initial entropy $H_{0}$.

Our starting point is the following uniformly ellipticity property of the matrix $\left(\bar{a}_{i j}\right)$, cf. Proposition 4 of [1]. 
Lemma 3.1. There exists a constant $K$, depending only on $\gamma$ and $M_{0}, E_{0}, H_{0}$, such that

$$
\sum_{1 \leq i, j \leq 3} \bar{a}_{i j}(t, v) \xi_{i} \xi_{j} \geq K\left(1+|v|^{2}\right)^{\gamma / 2}|\xi|^{2}, \quad \forall \xi \in \mathbb{R}^{3} .
$$

Remark 3.2. Although the ellipticity of $\left(a_{i j}\right)$ was proved in [11] under the hard potential case $\gamma>0$, it's still true for $\gamma=0$, the Maxwellian case. This can be seen in the proof of Proposition 4 of [11].

Lemma 3.3. There exists a constant $B$, depending only on the Gevrey index $\sigma>1$, such that for all multi-indices $\beta$ with $|\beta| \geq 2$ and all $g, h \in L_{\gamma}^{2}\left(\mathbb{R}^{3}\right)$,

$$
\int_{\mathbb{R}^{3}}\left(\partial^{\beta} \bar{a}_{i j}(t, v)\right) g(v) h(v) d v \leq C\|g\|_{L_{\gamma}^{2}}\|h\|_{L_{\gamma}^{2}}\left[G_{\sigma}(f(t))\right]_{\beta-2}, \quad \forall t>0,
$$

where $\left[G_{\sigma}(f(t))\right]_{\beta-2}=\left\{\left\|\partial^{\beta-2} f(t)\right\|_{L^{2}}+B^{|\beta|-2}[(|\beta|-2) !]^{\sigma}\right\}$.

Proof For $\sigma>1$, there exists a function $\psi \in G_{0}^{\sigma}\left(\mathbb{R}^{3}\right)$ compact support in $\left\{v \in \mathbb{R}^{3}|| v \mid \leq 2\right\}$, satisfying $\psi(v)=1$ on the ball $\left\{v \in \mathbb{R}^{3}|| v \mid \leq 1\right\}$, and moreover for some constant $L>4$ depending only on $\sigma$,

$$
\sup \left|\partial^{\lambda} \psi\right| \leq L^{|\lambda|}(|\lambda| !)^{\sigma}, \quad \forall \lambda
$$

For the construction of $\psi$, see [16] for example. Write $a_{i j}=\psi a_{i j}+(1-\psi) a_{i j}$. Then $\bar{a}_{i j}=\left(\psi a_{i j}\right) * f+\left[(1-\psi) a_{i j}\right] * f$, and hence

$$
\partial^{\beta} \bar{a}_{i j}=\left[\partial^{\tilde{\beta}}\left(\psi a_{i j}\right)\right] *\left(\partial^{\beta-\tilde{\beta}} f\right)+\left\{\partial^{\beta}\left[(1-\psi) a_{i j}\right]\right\} * f,
$$

where $\tilde{\beta}$ is an arbitrary multi-index satisfying $\tilde{\beta} \leq \beta$ and $|\tilde{\beta}|=2$. We firstly treat the term $\left[\partial^{\tilde{\beta}}\left(\psi a_{i j}\right)\right] *\left(\partial^{\beta-\tilde{\beta}} f\right)$. It is easy to verify that for all $\tilde{\beta}$ with $|\tilde{\beta}|=2$,

$$
\left|\left(\partial^{\tilde{\beta}} a_{i j}\right)\left(v-v_{*}\right)\right| \leq C\left|v-v_{*}\right|^{\gamma},
$$

from which, we can compute

$$
\begin{gathered}
\left|\left[\partial^{\tilde{\beta}}\left(\psi a_{i j}\right)\right] *\left(\partial^{\beta-\tilde{\beta}} f\right)(v)\right|=\left|\int_{\mathbb{R}^{3}}\left[\partial^{\tilde{\beta}}\left(\psi a_{i j}\right)\right]\left(v-v_{*}\right) \cdot\left(\partial^{\beta-\tilde{\beta}} f\right)\left(v_{*}\right) d v_{*}\right| \\
\leq C \int_{\left\{\left|v_{*}-v\right| \leq 2\right\}}\left|\left(\partial^{\beta-\tilde{\beta}} f\right)\left(v_{*}\right)\right| d v_{*} \\
\leq C\left\|\partial^{\beta-2} f(t)\right\|_{L^{2}}
\end{gathered}
$$

Next the term $\left\{\partial^{\beta}\left[(1-\psi) a_{i j}\right]\right\} * f$, we use Leibniz's formula to get

$$
\begin{gathered}
\left|\left(\partial^{\beta}\left[(1-\psi) a_{i j}\right]\right) * f(v)\right| \\
=\left|\sum_{0 \leq|\lambda| \leq|\beta|} C_{\beta}^{\lambda} \int_{\mathbb{R}^{3}}\left[\partial^{\beta-\lambda}(1-\psi)\right]\left(v-v_{*}\right) \cdot\left(\partial^{\lambda} a_{i j}\right)\left(v-v_{*}\right) \cdot f\left(t, v_{*}\right) d v_{*}\right| \\
\leq\left|\sum_{0 \leq|\lambda| \leq|\beta|-1} C_{\beta}^{\lambda} \int_{\left\{1 \leq\left|v_{*}-v\right| \leq 2\right\}}\left(\partial^{\beta-\lambda} \psi\right)\left(v-v_{*}\right) \cdot\left(\partial^{\lambda} a_{i j}\right)\left(v-v_{*}\right) \cdot f\left(t, v_{*}\right) d v_{*}\right| \\
+\left|\int_{\left\{\left|v_{*}-v\right| \geq 1\right\}}[(1-\psi)]\left(v-v_{*}\right) \cdot\left(\partial^{\beta} a_{i j}\right)\left(v-v_{*}\right) \cdot f\left(t, v_{*}\right) d v_{*}\right| \\
=J_{1}+J_{2} .
\end{gathered}
$$

In view of (2), we can find a constant $\tilde{C}$, such that for all multi-indices $\lambda$,

$$
\left|\left(\partial^{\lambda} a_{i j}\right)\left(v-v_{*}\right)\right| \leq \tilde{C}^{|\lambda|}|\lambda| ! \text { for } 1 \leq\left|v_{*}-v\right| \leq 2 .
$$

And for all $\beta$ with $|\beta| \geq 2$,

$$
\left|\left(\partial^{\beta} a_{i j}\right)\left(v-v_{*}\right)\right| \leq \tilde{C}^{|\beta|}|\beta| !\left(1+\left|v_{*}\right|^{\gamma}+|v|^{\gamma}\right) \quad \text { for }\left|v_{*}-v\right| \geq 1 \text {. }
$$


These along with (23) give the upper bound of $J_{1}$ and $J_{2}$,

$$
J_{1} \leq L^{|\beta|}(|\beta| !)^{\sigma} \cdot\|f(t)\|_{L^{1}} \sum_{0 \leq|\lambda| \leq|\beta|-1}\left(\frac{\tilde{C}}{L}\right)^{|\lambda|} ; \quad J_{2} \leq 2 \tilde{C}^{|\beta|}(|\beta| !)^{\sigma} \cdot\|f(t)\|_{L_{\gamma}^{1}}\left(1+|v|^{\gamma}\right) .
$$

By taking $L$ large enough such that $L \geq 2 \tilde{C}$, then we get

$$
J_{1}+J_{2} \leq C\|f(t)\|_{L_{\gamma}^{1}} L^{|\beta|}(|\beta| !)^{\sigma}\left(1+|v|^{2}\right)^{\gamma / 2} .
$$

This along with the fact $\|f(t)\|_{L_{\gamma}^{1}} \leq M_{0}+2 E_{0}$ gives at once

$$
\left|\left(\partial_{v}^{\beta}\left[(1-\psi) a_{i j}\right]\right) * f(v)\right| \leq J_{1}+J_{2} \leq C L^{|\beta|}(|\beta| !)^{\sigma}\left(1+|v|^{2}\right)^{\gamma / 2} .
$$

Now we choose a constant $B$ such that $L^{|\beta|}(|\beta| !)^{\sigma} \leq B^{|\beta|}[(|\beta|-2) !]^{\sigma}$. From the above inequality it follows immediately that

$$
\left|\left(\partial_{v}^{\beta}\left[(1-\psi) a_{i j}\right]\right) * f(v)\right| \leq C B^{|\beta|-2}[(|\beta|-2) !]^{\sigma}\left(1+|v|^{2}\right)^{\gamma / 2} .
$$

Combining the estimate on the term $\left[\partial^{2}\left(\psi a_{i j}\right)\right] *\left(\partial^{|\beta|-2} f\right)$, one has

$$
\begin{gathered}
\left|\partial^{\beta} \bar{a}_{i j}(v)\right| \leq C\left\{\left\|\partial^{\beta-2} f(t)\right\|_{L^{2}}+B^{|\beta|-2}[(|\beta|-2) !]^{\sigma} \cdot\left(1+|v|^{2}\right)^{\gamma / 2}\right\} \\
\leq C\left[G_{\sigma}(f(t))\right]_{\beta-2} \cdot\left(1+|v|^{2}\right)^{\gamma / 2} .
\end{gathered}
$$

Together with Cauchy's inequality, we get the desired inequality.

In quite similar argument, we have the following

Lemma 3.4. For all multi-indices $\beta$ with $|\beta| \geq 0$ and all $g, h \in L_{\gamma}^{2}\left(\mathbb{R}^{3}\right)$, one has

$$
\int_{\mathbb{R}^{3}}\left(\partial^{\beta} \bar{c}(t, v)\right) g(v) h(v) d v \leq C\|g\|_{L_{\gamma}^{2}}\|h\|_{L_{\gamma}^{2}} \cdot\left[G_{\sigma}(f(t))\right]_{\beta}, \quad \forall t \geq 0 .
$$

The rest of the paper is devoted to

Proof of Lemma 2.2. Let $b_{j}=\partial_{v_{i}} a_{i j}(v)=-2|v|^{\gamma} v_{j}$. Then we have the following relation

$$
\partial_{v_{i}} \bar{a}_{i j}(v)=\bar{b}_{j}(v), \quad \partial_{v_{j}} \bar{b}_{j}=\bar{c} .
$$

Since $f$ satisfies $\partial_{t} f=\bar{a}_{i j} \partial_{v_{i} v j} f-\bar{c} f$, then direct verification shows

$$
\begin{aligned}
\frac{d}{d t}\left\|\partial^{\mu} f(t)\right\|_{L^{2}}^{2} & =2 \int_{\mathbb{R}^{3}}\left[\partial_{t} \partial^{\mu} f(t, v)\right] \cdot\left[\partial^{\mu} f(t, v)\right] d v \\
= & 2 \int_{\mathbb{R}^{3}}\left[\partial^{\mu}\left(\bar{a}_{i j} \partial_{v_{i} v_{j}} f-\bar{c} f\right)\right] \cdot\left[\partial^{\mu} f(t, v)\right] d v .
\end{aligned}
$$

Moreover, using Leibniz's formula on the term $\partial^{\mu}\left(\bar{a}_{i j} \partial_{v_{i} v_{j}} f-\bar{c} f\right)$, one has

$$
\begin{aligned}
\partial_{t}\left\|\partial^{\mu} f(t)\right\|_{L^{2}}^{2}= & 2 \int_{\mathbb{R}^{3}} \bar{a}_{i j}\left(\partial_{v_{i} v_{j}} \partial^{\mu} f\right) \cdot\left(\partial^{\mu} f\right) d v \\
& +2 \sum_{|\beta|=1} C_{\mu}^{\beta} \int_{\mathbb{R}^{3}}\left(\partial^{\beta} \bar{a}_{i j}\right)\left(\partial_{v_{i} v_{j}} \partial^{\mu-\beta} f\right) \cdot\left(\partial^{\mu} f\right) d v \\
& +2 \sum_{2 \leq|\beta| \leq|\mu|} C_{\mu}^{\beta} \int_{\mathbb{R}^{3}}\left(\partial^{\beta} \bar{a}_{i j}\right)\left(\partial_{v_{i} v_{j}} \partial^{\mu-\beta} f\right) \cdot\left(\partial^{\mu} f\right) d v \\
& -2 \sum_{0 \leq|\beta| \leq|\mu|} C_{\mu}^{\beta} \int_{\mathbb{R}^{3}}\left(\partial^{\mu-\beta} \bar{c}\right)\left(\partial^{\beta} f\right) \cdot\left(\partial^{\mu} f\right) d v \\
= & (I)+(I I)+(I I I)+(I V) .
\end{aligned}
$$

We shall proceed to treat the above terms by the following steps. 


\section{Step 1. Upper bound for the term $(I)$.}

Integrating by parts, one has

$$
\begin{aligned}
(I) & =-2 \int_{\mathbb{R}^{3}} \bar{a}_{i j}\left(\partial_{v_{j}} \partial^{\mu} f\right) \cdot\left(\partial_{v_{i}} \partial^{\mu} f\right) d v-2 \int_{\mathbb{R}^{3}} \bar{b}_{j}\left(\partial_{v_{j}} \partial^{\mu} f\right) \cdot\left(\partial^{\mu} f\right) d v \\
& =(I)_{1}+(I)_{2} .
\end{aligned}
$$

The ellipticity property (22) of $\left(a_{i j}\right)$ gives that

$$
(I)_{1} \leq-2 K \int_{\mathbb{R}^{3}}\left|\nabla_{v} \partial^{\mu} f\right|^{2}\left(1+|v|^{2}\right)^{\gamma / 2} d v=-2 K\left\|\nabla_{\nu} \partial^{\mu} f(t)\right\|_{L_{\gamma}^{2}}^{2} .
$$

For the term $(I)_{2}$, integrating by parts again, we have

$$
(I)_{2}=-(I)_{2}+2 \int_{\mathbb{R}^{3}} \bar{c}\left(\partial^{\mu} f\right) \cdot\left(\partial^{\mu} f\right) d v .
$$

This along with the fact $|\bar{c}(v)| \leq C\|f(t)\|_{L_{\gamma}^{1}}\left(1+|v|^{2}\right)^{\gamma / 2} \leq C\left(1+|v|^{2}\right)^{\gamma / 2}$ shows immediately

$$
(I)_{2} \leq C\left\|\partial^{\mu} f(t)\right\|_{L_{\gamma}^{2}}^{2} \leq C\left\|\nabla_{\nu} \partial^{\mu-1} f(t)\right\|_{L_{\gamma}^{2}}^{2} .
$$

Combining these, we get the estimate on the term $(I)$, that is

$$
(I) \leq-2 K\left\|\nabla_{\nu} \partial^{\mu} f(t)\right\|_{L_{\gamma}^{2}}^{2}+C\left\|\nabla_{\nu} \partial^{\mu-1} f(t)\right\|_{L_{\gamma}^{2}}^{2} .
$$

\section{Step 2. Upper bound for the term (II).}

Recall $(I I)=2 \sum_{|\beta|=1} C_{\mu}^{\beta} \int_{\mathbb{R}^{3}}\left(\partial^{\beta} \bar{a}_{i j}\right)\left(\partial_{v_{i} v_{j}} \partial^{\mu-\beta} f\right) \cdot\left(\partial^{\mu} f\right) d v$. Integrating by parts, we get

$$
\begin{gathered}
(I I) \quad=-2 \sum_{|\beta|=1} C_{\mu}^{\beta} \int_{\mathbb{R}^{3}}\left(\partial^{\beta} \bar{b}_{j}\right)\left(\partial_{v_{j}} \partial^{\mu-\beta} f\right) \cdot\left(\partial^{\mu} f\right) d v \\
-2 \sum_{|\beta|=1} C_{\mu}^{\beta} \int_{\mathbb{R}^{3}}\left(\partial^{\beta} \bar{a}_{i j}\right)\left(\partial_{v_{j}} \partial^{\mu-\beta} f\right) \cdot\left(\partial_{v_{i}} \partial^{\mu} f\right) d v \\
=(I I)_{1}+(I I)_{2} .
\end{gathered}
$$

Note $\left|\partial^{\beta} \bar{b}_{j}(t, v)\right| \leq C\left(1+|v|^{2}\right)^{\gamma / 2}$ for any $\beta$ with $|\beta|=1$ and hence

$$
(I I)_{1} \leq C|\mu| \cdot\left\|\nabla_{\nu} \partial^{\mu-\beta} f(t)\right\|_{L_{\gamma}^{2}}\left\|\partial^{\mu} f(t)\right\|_{L_{\gamma}^{2}} \leq C|\mu| \cdot\left\|\nabla_{\nu} \partial^{\mu-1} f(t)\right\|_{L_{\gamma}^{2}}^{2} .
$$

For the term $(I I)_{2}$, noticing that $\mu=\beta+(\mu-\beta)$, it can be rewritten as the following form

$$
(I I)_{2}=-2 \sum_{|\beta|=1} C_{\mu}^{\beta} \int_{\mathbb{R}^{3}}\left(\partial^{\beta} \bar{a}_{i j}\right)\left(\partial_{v_{j}} \partial^{\mu-\beta} f\right) \cdot\left(\partial^{\beta} \partial_{v_{i}} \partial^{\mu-\beta} f\right) d v .
$$

Since $|\beta|=1$, we can integrate by parts to get

$$
\begin{aligned}
&(I I)_{2}= 2 \sum_{|\beta|=1} C_{\mu}^{\beta} \int_{\mathbb{R}^{3}}\left(\partial^{\beta} \bar{a}_{i j}\right)\left(\partial_{v_{j}} \partial^{\mu} f\right) \cdot\left(\partial_{v_{i}} \partial^{\mu-\beta} f\right) d v \\
&+2 \sum_{|\beta|=1} C_{\mu}^{\beta} \int_{\mathbb{R}^{3}}\left(\partial^{\beta+\beta} \bar{a}_{i j}\right)\left(\partial_{v_{j}} \partial^{\mu-\beta} f\right) \cdot\left(\partial_{v_{i}} \partial^{\mu-\beta} f\right) d v \\
&=-(I I)_{2}+2 \sum_{|\beta|=1} C_{\mu}^{\beta} \int_{\mathbb{R}^{3}}\left(\partial^{\beta+\beta} \bar{a}_{i j}\right)\left(\partial_{v_{j}} \partial^{\mu-\beta} f\right) \cdot\left(\partial_{v_{i}} \partial^{\mu-\beta} f\right) d v .
\end{aligned}
$$

Hence

$$
(I I)_{2}=\sum_{|\beta|=1} C_{\mu}^{\beta} \int_{\mathbb{R}^{3}}\left(\partial^{\beta+\beta} \bar{a}_{i j}\right)\left(\partial_{v_{j}} \partial^{\mu-\beta} f\right) \cdot\left(\partial_{v_{i}} \partial^{\mu-\beta} f\right) d v .
$$

This along with the fact $\left|\partial^{\beta+\beta} \bar{a}_{i j}(v)\right| \leq C\left(1+|v|^{2}\right)^{\gamma / 2}$ for all $\beta$ with $|\beta|=1$ shows at once

$$
(I I)_{2} \leq C \sum_{|\beta|=1} C_{\mu}^{\beta} \cdot\left\|\nabla_{\nu} \partial^{\mu-\beta} f\right\|_{L_{\gamma}^{2}}^{2} \leq C|\mu| \cdot\left\|\nabla_{\nu} \partial^{\mu-1} f\right\|_{L_{\gamma}^{2}}^{2} .
$$

Combining these, we obtain

$$
(I I) \leq C|\mu| \cdot\left\|\nabla_{\nu} \partial^{|\mu|-1} f\right\|_{L_{\gamma}^{2}}^{2}
$$


Step 3. Upper bound for the term $(I I I)$ and $(I V)$ and the conclusion.

Recall $(I I I)=2 \sum_{2 \leq|\beta| \leq|\mu|} C_{\mu}^{\beta} \int_{\mathbb{R}^{3}}\left(\partial^{\beta} \bar{a}_{i j}\right)\left(\partial_{v_{i} v_{j}} \partial^{\mu-\beta} f\right) \cdot\left(\partial^{\mu} f\right) d v$ and

$$
(I V)=-2 \sum_{0 \leq|\beta| \leq|\mu|} C_{\mu}^{\beta} \int_{\mathbb{R}^{3}}\left(\partial^{\mu-\beta} \bar{c}\right)\left(\partial^{\beta} f\right) \cdot\left(\partial^{\mu} f\right) d v .
$$

By virtue of Lemma 3.3 and lemma 3.4, it follows that

$$
\begin{aligned}
& (I I I) \leq C \sum_{2 \leq|\beta| \leq|\mu|} C_{\mu}^{\beta}\left\|\partial_{v_{i} v_{j}} \partial^{\mu-\beta} f(t)\right\|_{L_{\gamma}^{2}} \cdot\left\|\partial^{\mu} f(t)\right\|_{L_{\gamma}^{2}}\left[G_{\sigma}(f(t))\right]_{\beta-2} \\
& \leq C \sum_{2 \leq|\beta| \leq|\mu|} C_{\mu}^{\beta}\left\|\nabla_{\nu} \partial^{\mu-\beta+1} f(t)\right\|_{L_{\gamma}^{2}} \cdot\left\|\nabla_{\nu} \partial^{\mu-1} f(t)\right\|_{L_{\gamma}^{2}}\left[G_{\sigma}(f(t))\right]_{\beta-2},
\end{aligned}
$$

and

$$
(I V) \leq C \sum_{0 \leq|\beta| \leq|\mu|} C_{\mu}^{\beta}\left\|\partial^{\beta} f(t)\right\|_{L_{\gamma}^{2}} \cdot\left\|\nabla_{\nu} \partial^{\mu-1} f(t)\right\|_{L_{\gamma}^{2}} \cdot\left[G_{\sigma}(f(t))\right]_{\mu-\beta} .
$$

Combination of (24)-(27) gives the desired inequality

$$
\begin{gathered}
\frac{d}{d t} \quad\left\|\partial^{\mu} f(t)\right\|_{L^{2}}^{2}+C_{1}\left\|\nabla_{v} \partial^{\mu} f(t)\right\|_{L_{\gamma}^{2}}^{2} \leq C_{2}|\mu|^{2}\left\|\nabla_{v} \partial^{\mu-1} f(t)\right\|_{L_{\gamma}^{2}}^{2} \\
+C_{2} \sum_{2 \leq|\beta| \leq|\mu|} C_{\mu}^{\beta}\left\|\nabla_{v} \partial^{\mu-\beta+1} f(t)\right\|_{L_{\gamma}^{2}} \cdot\left\|\nabla_{v} \partial^{\mu-1} f(t)\right\|_{L_{\gamma}^{2}} \cdot\left[G_{\sigma}(f(t))\right]_{\beta-2} \\
+C_{2} \sum_{0 \leq|\beta| \leq|\mu|} C_{\mu}^{\beta}\left\|\partial^{\beta} f(t)\right\|_{L_{\gamma}^{2}} \cdot\left\|\nabla_{v} \partial^{\mu-1} f(t)\right\|_{L_{\gamma}^{2}} \cdot\left[G_{\sigma}(f(t))\right]_{\mu-\beta},
\end{gathered}
$$

where $C_{1}, C_{2}$ are two constants depending only on $M_{0}, E_{0}, H_{0}, \sigma$ and $\gamma$. This completes the proof of Lemma 2.2.

\section{REFERENCES}

[1] R. Alexandre, L. Desvillettes, C. Villani, B. Wennberg, Entropy dissipation and long-range interactions, Arch. Rational Mech. Anal. 152 (2000) 327-355.

[2] R. Alexandre, M. Safadi, Littlewood Paley decomposition and regularity issues in Boltzmann equation homogeneous equations. I. Non cutoff and Maxwell cases, M3AM (2005) 8-15.

[3] R. Alexandre, S. Ukai, Y. Morimoto, C.-J. Xu, T. Yang, Uncertainty principle and regularity for Boltzmann equation,

[4] H. Chen, W.-X. Li, C.-J. Xu. The Gevrey Hypoellipticity for linear and non-linear Fokker-Planck equations.

[5] H. Chen, L.Rodino, General theory of PDE and Gevrey class. General theory of partial differential equations and microlocal analysis (Trieste 1995), Pitman Res. Notes in Math. Ser., 349, Longman, Harlow, 6-81, (1996).

[6] Y.-M. Chen, L. Desvillettes, L.-B. He, Smoothing Effects for Classic Solutions of the Full Landau Equation. Preprint.

[7] M.Derridj, C.Zuily, Sur la régularité Gevrey des opérateurs de Hörmander. J.Math.Pures et Appl. 52 (1973), 309-336.

[8] L. Desvillettes, On asymptotics of the Boltzmann equation when the collisions become grazing Transp. Th. Stat. Phys. 21, 3(1992), 259-276.

[9] L. Desvillettes, G. Furioli, E. Terraneo. Propagation of Gevrey regularity for solutions of the Boltzmann equation for Maxwellian molecules. Preprint.

[10] L. Desvillettes, B. Wennberg. Smoothness of the solution of the spatially homogeneous Boltzmann equation without cutoff. Comm. Partial Differential Equations 29 (2004), no. 1-2, 133-155.

[11] L. Desvillettes, C. Villani. On the Spartially Homogeneous Landau Equation for Hard Potentials, Part I: Existence, Uniqueness and Smoothness. Comm. Partial Differential Equations 25 (2000), no. 1-2, 179-259.

[12] M.Durand, Régularité Gevrey d'une classe d'opérateurs hypo-elliptiques. J.Math.Pures et Appl. 57 (1978), 323-360

[13] Y. Guo. The Landau Equation in a Periodic Box. Comm. Math. Phys. 231, 3 (2002), 391-434.

[14] Y. Morimoto and C.-J. Xu, Logarithmic Sobolev inequality and semi-linear Dirichlet problems for infinitely degenerate elliptic operators, Astérisque 284 (2003), 245-264. 
[15] Y. Morimoto, S. Ukai, C.-J. Xu, T. Yang, Regularity of solutions to the spatially homogeneous Boltzmann equation without Angular cutoff, preprint.

[16] L.Rodino, Linear partial differential operators in Gevrey class, World Scientific, Singapore, 1993.

[17] S. Ukai, Local solutions in Gevrey classes to the nonlinear Boltzmann equation without cutoff, Japan J. Appl. Math., 1(1984), no. 1, 141-156.

[18] C. Villani, On a new class of weak solutions to the spatially homogeneous Boltzmann and Landau equations, Arc. Rational Mech. Anal., 143 (1998), 273-307.

[19] C. Villani, On the spatially homogeneous Landau equations for Maxwellian molecules, Math. Models Methods Appl. Sci., 8 (1998), no. 6, 957-983, .

School of Mathematics and Statistics, Wuhan University, Wuhan 430072, P. R. China

E-mail address: chenhua@whu.edu.cn

School of Mathematics and Statistics, Wuhan University, Wuhan 430072, P. R. China

E-mail address: weixi.li@yahoo.com

School of Mathematics and Statistics, Wuhan University, Wuhan 430072, P. R. China

AND

Université de Rouen, UMR 6085-CNRS, MathématiQues, Avenue de l'Université, BR.12,

F76801 Saint Etienne du Rouvray, France

E-mail address: Chao-Jiang.Xu@univ-rouen.fr 